\title{
Thermodynamic limit for particle monodispersity: How narrow can a particle size distribution be?
}

\author{
Victor Burlakov and Alain Goriely \\ Mathematical Institute, Woodstock Road, Andrew Wiles Building, University of Oxford.
}

PACS 03.75. Hh - Static properties of condensates

PACS $05.70 . \mathrm{Np}$ - Interface and surface thermodynamics

PACS 64.60.-i-General studies of phase transitions

\begin{abstract}
A key problem in many emerging nanotechnologies is to create a narrow distribution of particles. Here, we prove the existence of a fundamental lower bound for the width of a particle size distribution (PSD) at equilibrium. This thermodynamic limit arises from the decrease of entropy for an ideal monodisperse distribution and is determined by the effective surface energy and temperature. The existence of this limit, provided that it is energetically favourable, implies that it is not possible to generate an arbitrarily narrow PSD through any processes that reaches a thermodynamic equilibrium. We show that if a narrow PSD is generated it will tend to broaden in time until it reaches an equilibrium PSD of Gaussian shape. Conversely, if the initial PSD is broad and free to evolve to its equilibrium shape, it will eventually acquire the same Gaussian shape and width. The effect of PSD broadening can be significant for functional materials built from nanoparticulate arrays. Our study provides the framework for understanding and describing this phenomenon.
\end{abstract}

Introduction. - A fundamental question in nanotechnology is to obtain nanoparticles of uniform size. Many high-performance functional materials and devices rely on arrays of nanoparticles as the active component [1-7]. In these systems, the width of the particle size distribution (PSD) plays a critical role [8-10]. Typically, the device functionality increases with the narrowness of the PSD [11-14]. Therefore, the ideal PSD should be uniform (zero width). A natural question is then: are there thermodynamics constraints that prevent the formation of an ideal PSD? Here, we obtain these constraints under the assumption that the particles are kept under thermodynamically controlled conditions. To do so, we consider small deviations around an ideal PSD and analyze the thermodynamic limit of uniform distributions of particles, which can be expressed in terms of the minimal achievable PSD width.

We assume the existence of a narrowing mechanism favoring the formation of identical particles, i.e. bringing their size distribution towards a uniform one. This can be achieved by passivation of particle surfaces so that the effective surface energy becomes negative $[15,16]$. For a negative surface energy and conserved number of particles, the energy minimum is reached when all the particles are identical. PSD narrowing implies that the configurational entropy of the particle ensemble decreases towards zero. Indeed, any permutation among identical particles does not generate a new distinguishable state and the configurational entropy of an ideal uniform PSD vanishes. Note that we do not consider the contribution of translational entropy since it does not change with a change of PSD width. We conclude that any deviation of the PSD from an ideal distribution will increase the particle ensemble entropy by an amount $\Delta S$. Therefore, there must be a balance between the narrowing tendency and the decrease of entropy.

PSD in spherical particles. - To illustrate this process, we start with an initial PSD made of an ensemble of $N$ spherical particles with an initial distribution with mean radius $R_{0}$. This ensemble of particles is kept at constant temperature and pressure and we assume that the particles can exchange mass. Assuming that the mass dissolved in the medium can be neglected, any mass exchange between the particles may broaden the PSD under the constraint that their total mass is conserved. We are interested in the equilibrium width of the PSD acquired by the particles. Consider a PSD described by the function 
$f(R)$ with mass constraint normalized such that

$$
\int_{0}^{\infty} f(R) \mathrm{d} R=1
$$

Then, the excess entropy $\Delta S$ can be expressed as

$$
\frac{\Delta S}{k_{\mathrm{B}}}=N \int_{0}^{\infty} f(R) \ln (f(R)) \mathrm{d} R
$$

During broadening some particles increase in size while other decrease in size. Therefore, the particles' total energy is changed by an amount $\Delta E$. Since the total mass is conserved, the change in energy is due to a change in the total surface area and is expressed as

$$
\begin{aligned}
\Delta E & =-4 \pi \gamma N \int_{0}^{\infty}\left(R^{2}-R_{0}^{2}\right) f(R) \mathrm{d} R \\
& =4 \pi \gamma N R_{0}^{2}-4 \pi \gamma N \int_{0}^{\infty} R^{2} f(R) \mathrm{d} R .
\end{aligned}
$$

where $-\gamma<0$ is the effective surface energy per atom and all radii are measured in units of interatomic distance. The minus sign in front of the integral is chosen to reflect the assumption that there is a thermodynamic driving force towards identical particles.

It is convenient to express $\Delta E$ in terms of an average radius

$$
\widehat{R}=\int_{0}^{\infty} R f(R) \mathrm{d} R
$$

and expressing $R_{0}$ as $R_{0}=\widehat{R}+\delta$ :

$$
\begin{aligned}
\Delta E & =4 \pi \gamma N R_{0}^{2}-4 \pi \gamma N \int_{0}^{\infty} R^{2} f(R) \mathrm{d} R \\
& =4 \pi \gamma N(\widehat{R}+\delta)^{2}-4 \pi \gamma N \int_{0}^{\infty}(R-\widehat{R}+\widehat{R})^{2} f(R) \mathrm{d} R \\
& =4 \pi \gamma N\left(2 \widehat{R} \delta+\delta^{2}\right)-4 \pi \gamma N \int_{0}^{\infty}(R-\widehat{R})^{2} f(R) \mathrm{d} R
\end{aligned}
$$

Next, we use the conservation of total volume:

$$
\frac{4 \pi}{3} N R_{0}^{3}=\frac{4 \pi}{3} \int_{0}^{\infty} R^{3} f(R) \mathrm{d} R,
$$

and expand both sides of this expression around $\widehat{R}$. That is,

$$
\begin{aligned}
R_{0}^{3}= & (\widehat{R}+\delta)^{3} \\
= & \int_{0}^{\infty}(R-\widehat{R}+\widehat{R})^{3} f(R) \mathrm{d} R \\
= & \int_{0}^{\infty}(R-\widehat{R})^{3} f(R) \mathrm{d} R \\
& +3 \widehat{R} \int_{0}^{\infty}(R-\widehat{R})^{2} f(R) \mathrm{d} R+\widehat{R}^{3} .
\end{aligned}
$$

Hence, we have

$$
\begin{aligned}
3 \widehat{R}^{2} \delta+3 \widehat{R} \delta^{2}= & \int_{0}^{\infty}(R-\widehat{R})^{3} f(R) \mathrm{d} R \\
& +3 \widehat{R} \int_{0}^{\infty}(R-\widehat{R})^{2} f(R) \mathrm{d} R .
\end{aligned}
$$

This last expression can be further simplified by assuming that $\delta \ll \widehat{R}$ and

$$
\int_{0}^{\infty}(R-\widehat{R})^{3} f(R) \mathrm{d} R \ll 3 \widehat{R} \int_{0}^{\infty}(R-\widehat{R})^{2} f(R) \mathrm{d} R .
$$

This approximation will be justified a posteriori. Using both assumptions we have

$$
\widehat{R} \delta \approx \int_{0}^{\infty}(R-\widehat{R})^{2} f(R) \mathrm{d} R
$$

We can now use this relationship to simplify the expression (5) for $\Delta E$ :

$$
\Delta E \approx 4 \pi \gamma N \int_{0}^{\infty}(R-\widehat{R})^{2} f(R) \mathrm{d} R,
$$

where we have, again, neglected terms of order $\mathcal{O}\left(\delta^{2}\right)$.

The approximate excess free energy is then given by

$$
\begin{aligned}
\Delta G & =\Delta E-T \Delta S \\
& =N \int_{0}^{\infty}\left[4 \pi \gamma(R-\widehat{R})^{2}+k_{\mathrm{B}} T \log f(R)\right] f(R) \mathrm{d} R .
\end{aligned}
$$

The minimization of $\mathcal{G}[f]=\Delta G / N$ with respect to $f$ provides the equilibrium distribution. This minimization is carried out explicitly in the Appendix where it is shown that if the width $\sigma$ of the distribution is much smaller than the ideal radius $R_{0}$, the exact distribution on the positive real axis can be replaced by the Gaussian distribution

$$
f_{\text {app }}(R)=\frac{1}{\sigma \sqrt{2 \pi}} \exp \left(-\frac{(R-\widehat{R})^{2}}{2 \sigma^{2}}\right)
$$

with

$$
\widehat{R}=R_{0}\left(1-\frac{\sigma^{2}}{R_{0}^{2}}\right), \quad \sigma=\sqrt{\frac{k_{\mathrm{B}} T}{8 \pi \gamma}} .
$$

This approximation is valid for $\epsilon \ll 1$ where

$$
\epsilon=\frac{\sigma}{R_{0}}=\sqrt{\frac{k_{\mathrm{B}} T}{8 \pi \gamma R_{0}^{2}}} .
$$

Kinetic Monte Carlo Modeling. - We complement our theoretical analysis with a Monte Carlo simulation of this process. We assume that a particle can emit a unit of volume $a^{3}$ (one atom) with a probability $P(R)$ per unit surface area determined by the Gibbs-Thomson concentration $n_{\mathrm{GT}}$ and the number of surface atoms

$$
P_{\text {out }}(R)=4 \pi\left(\frac{R}{a}\right)^{2} K n_{\mathrm{GT}},
$$

where $K$ is the kinetic constant. Similarly, we define an absorption probability via atomic concentration in solution $n$ as

$$
P_{\text {in }}(R)=4 \pi\left(\frac{R}{a}\right)^{2} K n .
$$



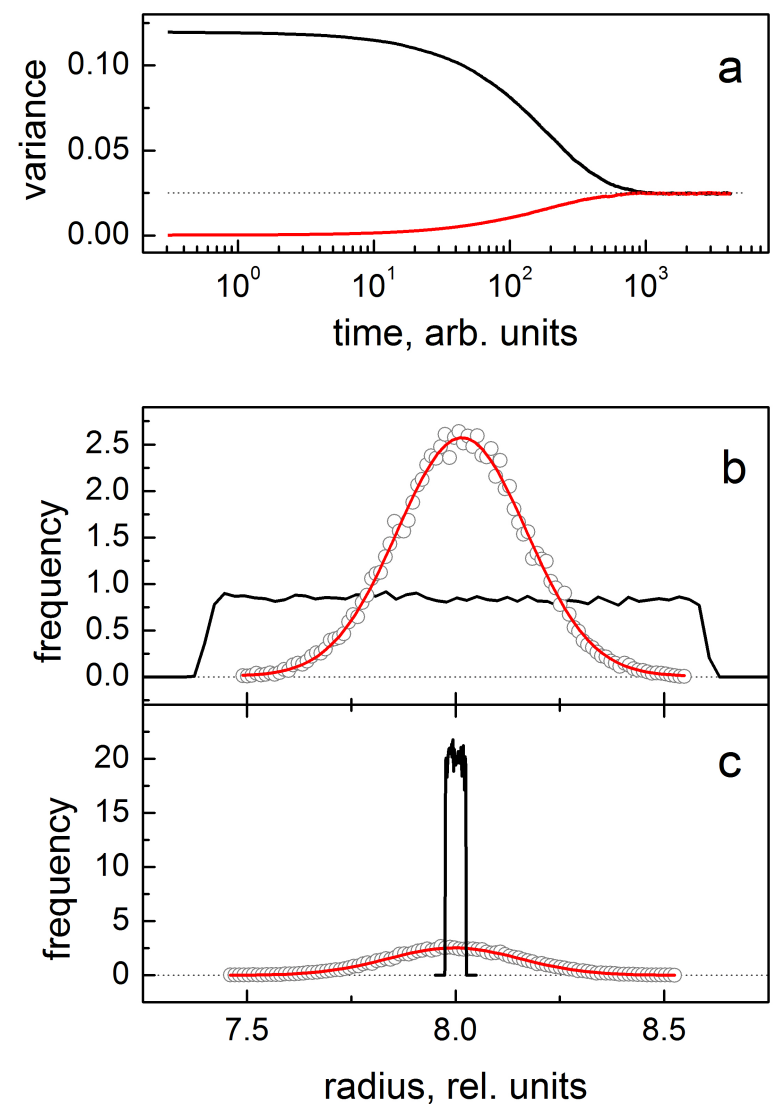

Fig. 1: Monte Carlo simulations for $\gamma=0.05 k_{b} T$. a: Evolution of the variance for two initial distributions. b: Evolution of a broad initial distribution towards the equilibrium distribution together with the Gaussian approximation. c: Evolution of a narrow initial distribution towards the equilibrium distribution together with the Gaussian approximation. For all case, the dotted line is the approximation given by (27).

The net flux is

$$
4 \pi R^{2} \frac{\mathrm{d} R}{\mathrm{~d} t}=a^{3}\left(P_{\mathrm{in}}-P_{\mathrm{out}}\right)=a 4 \pi R^{2} K\left(n-n_{\mathrm{GT}}\right) .
$$

This expression is similar to the one obtained in the classical problem of ripening in the Wagner approximation, i.e. interface-controlled ripening [17]. The concentration $n$ is defined as usual, by means of atomic number conservation

$$
\sum R^{2} n_{\mathrm{GT}}(R)=\sum R^{2} n
$$

That is, we have

$$
n\left\langle R^{2}\right\rangle=\left\langle R^{2} n_{\mathrm{GT}}(R)\right\rangle
$$

which implies

$$
\begin{aligned}
n & =\frac{\left\langle R^{2} n_{\mathrm{GT}}(R)\right\rangle}{\left\langle R^{2}\right\rangle} \\
& =\frac{1}{\left\langle R^{2}\right\rangle} n_{0} \exp \left(-\frac{\epsilon_{\mathrm{coh}}}{k_{b} T}\right)\left\langle R^{2} \exp \left(\frac{2 \gamma}{R k_{b} T}\right)\right\rangle,
\end{aligned}
$$

where we have used the expression for Gibbs-Thomson concentration

$$
n_{\mathrm{GT}}(R)=\exp \left(-\frac{\epsilon_{\mathrm{coh}}}{k_{b} T}+\frac{2 \gamma}{R k_{b} T}\right) .
$$

The probabilities in (18) can be rewritten omitting the factor $n_{0} \exp \left(-\frac{\epsilon_{\mathrm{coh}}}{k_{b} T}\right)$ as

$$
\begin{aligned}
& P_{\text {in }}\left(R_{j}\right)=4 \pi \frac{R_{j}^{2}}{\left\langle R^{2}\right\rangle}\left\langle R^{2} \exp \frac{2 \gamma a}{R k_{b} T}\right\rangle, \\
& P_{\text {out }}\left(R_{j}\right)=4 \pi{R_{j}}^{2} \exp \frac{2 \gamma a}{R_{j} k_{b} T} .
\end{aligned}
$$

Note that these probabilities preserve the total number of atoms. We generate a random number Ran evenly spread between 0 and 1 , and we chose the reaction and time steps following the usual for kinetic Monte Carlo method. Then, we change the corresponding particle radius (measured in $a$ units) to

$$
\begin{aligned}
R_{i}(t+\Delta t) & =\left(\left(R_{i}(t)\right)^{3} \pm \frac{3}{4 \pi}\right)^{1 / 3} \\
& \approx R_{i}(t) \pm \frac{1}{4 \pi\left(R_{i}(t)\right)^{2}} \\
t_{i+1}-t_{i}= & -\frac{\ln (\operatorname{Ran})}{\sum_{j}\left(m P_{\mathrm{in}}\left(R_{j}\right)+P_{\mathrm{out}}\left(R_{j}\right)\right)}
\end{aligned}
$$

where $m$ is the number of atoms in the vapor phase. The sign \pm in these expressions is determined by the corresponding reaction (either by emission or by absorption of atoms). In our computations, we have chosen boxcar initial distributions with two significantly different values of the initial variance. In the course of the simulations these distributions converge to a single thermodynamically stable Gaussian PSD with the same variance (see Fig. 1). The theoretical variance of this PSD is given by

$$
\left\langle\left(R-R_{0}\right)^{2}\right\rangle=\sigma^{2}=\frac{k_{\mathrm{B}} T}{8 \pi \gamma}
$$

This expression is valid for a broad range of surface energy and temperature values as shown in Fig. 2, even for the value of $\gamma=0.0125 \mathrm{eV}$, which gives a variance of 0.1 , corresponding to a width of the PSD close to 0.74 in relative units. As this result holds for any particle ensemble, it implies that the radius variation may be quite significant for an ensemble with small mean radius. The fact that the variance does not depend on the mean radius can be understood a posteriori on dimensional grounds. Assuming that the process mostly depends on the surface energy and temperature, a length scale $\sim k_{\mathrm{B}} T / \gamma$ naturally appears in the problem as a balance between a binding term (surface energy) and a spreading term related to entropy (temperature).

Note that we give here the time scale in arbitrary units. In fact the KMC approach can be further developed to estimate the characteristic time scale for stability of ideal 


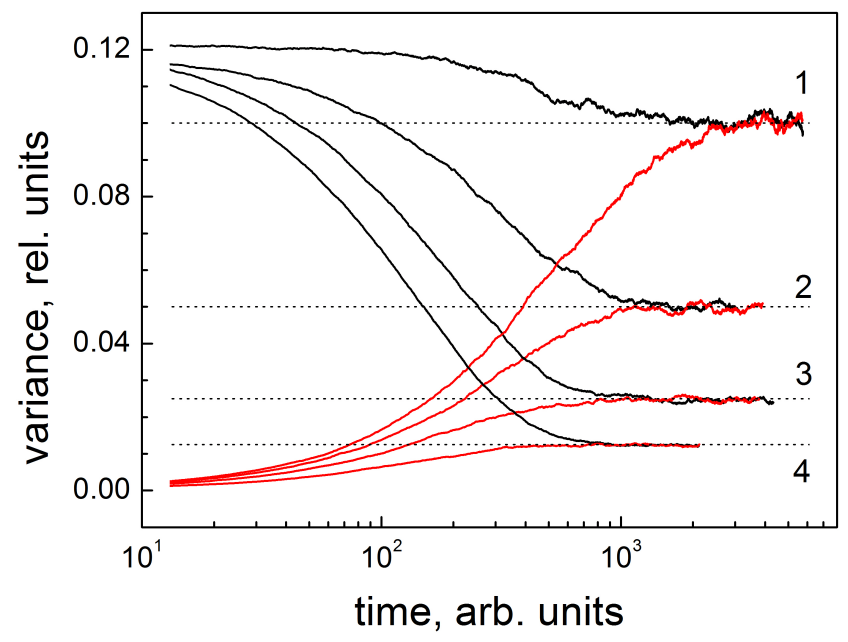

Fig. 2: Variation of the variance for various values of $\gamma$ for curve $1(\gamma=-0.0125), 2(\gamma=-0.025), 3(\gamma=-0.05), 4(\gamma=-0.1)$, all in units of $k_{\mathrm{B}} T$. The dotted lines are given by the Gaussian approximation $\sigma^{2}$.

monodisperse ensemble, or for transforming a broader PSD down to an equilibrium one if the values of surface reaction rates $K$ are available. It can also be extended to simulate diffusion-controlled systems at higher computational costs.

The above analysis is based on the assumption that the total mass of atoms dissolved in surrounding medium is negligible compared to the total mass of particles, which is consistent with a description of the particle system using a canonical ensemble. This is a common and realistic assumption in describing the processes of particles ripening. It implies that the solution (or vapor) volume is not very large. It is informative to analyze the opposite case when the solution volume is large enough such that the dissolved material mass is much larger than the mass of the particles. In this case, the atomic chemical potential $\mu$ is fixed by the chemical potential $\mu_{S}$ of the atoms in the solution. Therefore, the system should now be treated using the grand canonical ensemble. Here we have to discriminate between the two extreme cases when: 1) particles are far away from each other such that the condition of fixed $\mu=\mu_{S}$ is applicable to each individual particle; or 2) particles form a compact subsystem and the condition about the equivalence of chemical potentials has to be written with respect to the average chemical potential of the particles, namely $\langle\mu\rangle=\mu_{S}$.

The first case is trivial: fixed $\mu$ for a particle means that its radius is fixed, suggesting that any size fluctuations are greatly suppressed, and all particles would keep the same radius determined by $\mu_{S}$. The second case gives a strikingly different result. Any size fluctuation in the compact subsystem results in a decrease of $\langle\mu\rangle$ simply because $\mu \sim 1 / R$ (where $R$ is the particle radius) and a decrease in $\mu$ due to a decrease in $R$ is higher than an increase in $\mu$ due to the same increase in $R$. A broadening of the PSD due to a fluctuation implies an intake of atoms from the surrounding medium to compensate for the decrease in $\langle\mu\rangle$. This intake decreases the system's energy since both cohesive and surface atomic energies are negative. Therefore, the process will progress until it is energetically favorable leading to a further mass accumulation by the particles and broadening of their PSD.

The two extreme cases that we have considered suggest a way of storing monodisperse particles. According to the first case, it is preferable to store particles as a dilute suspension in the bulk of a solvent with a properly chosen concentration of the corresponding atomic species. However, if a few particles come close enough to form a compact subsystem, they will develop, locally, a broadening of their size distribution. An alternative way of storing monodisperse particles is to keep them as a compact system within a small solution volume to allow the formation of a thermodynamically stable PSD.

Another major approximation in the present study is that the the particles are all considered to be spherical. It is a reasonable approximation for colloidal particles or for amorphous solid particles. However, in the case of crystalline particles this approximation can be violated requiring a much more sophisticated analysis of size and shape evolution. In particular with surface passivation, the particles can develop facets resulting in a peculiar growth kinetics, including system's arrest in an intermediate state [18].

Conclusions.. - Our study demonstrates that configurational entropy provides a fundamental restriction for particles monodispersity. The fundamental reason for this theoretical limitation is that in an ideal monodisperse system, i.e. an ensemble of identical particles, the configurational entropy vanishes. Hence, a monodisperse system cannot be thermodynamically stable. The thermodynamic driving force for particle monodispersity is the surface energy, which can be made negative by surface passivation, i.e. by using ligands binding strongly with the particle surfaces. The opposing force is due to the configurational entropy. Since, it is proportional to the system's temperature, it is intuitively clear that the variance of the particle size distribution should be proportional to the system temperature and inversely proportional to the surface energy. Hence the distribution width decreases (monodispersity increases) as the temperature-to-surface-energy decreases. An important result is that the fundamental limit for the PSD variance does not depend on the mean particle radius. Experimentally, our theory illustrates the importance of ligands in size stabilization, as ligands make the effective surface energy negative $[19,20]$. A general ruleof-thumb for monodispersity is that $R>20 \sigma$ [21]. Therefore, for $\gamma=k_{\mathrm{B}} T / 4$, our theory predicts that the radius must exceed 8 interatomic distances. The existence of the fundamental limit for monodispersity can be critically important for functional materials based on small particles, such as nanomaterials. 
Appendix: Minimization of the free energy. The problem is to minimize the functional

$$
\mathcal{G}[f]=N \int_{0}^{\infty}\left(4 \pi \gamma(R-\widehat{R})^{2}+k_{\mathrm{B}} T \ln f(R)\right) f(R) \mathrm{d} R,
$$

with the constraint

$$
\widehat{R}=\int_{0}^{\infty} R f(R) \mathrm{d} R
$$

We also require that the distribution is normalized. That is, we have the additional constraint

$$
1=\int_{0}^{\infty} f(R) \mathrm{d} R
$$

To solve this problem, we use the theory of Lagrange multipliers. To do so, we introduce the functionals

$$
\mathcal{C}_{1}[f]=\widehat{R}-\int_{0}^{\infty} R f(R) \mathrm{d} R,
$$

and

$$
\mathcal{C}_{2}[f]=1-\int_{0}^{\infty} f(R) \mathrm{d} R .
$$

The new functional to minimize is

$$
\mathcal{L}\left[f, q_{1}, q_{2}\right]=\mathcal{G}[f]+q_{1} \mathcal{C}_{1}[f]+q_{2} \mathcal{C}_{2}[f]
$$

where $q_{1}$ and $q_{2}$ are the Lagrange multipliers to be determined. Taking variations with respect to $f$ and $q_{i}$, gives the Euler-Lagrange equations for this problem:

$$
\begin{aligned}
& \left(4 \pi \gamma(R-\widehat{R})^{2}+k_{\mathrm{B}} T \ln f(R)\right)+k_{\mathrm{B}} T-q_{1} R-q_{2}=0 \\
& \widehat{R}=\int_{0}^{\infty} R f(R) \mathrm{d} R \\
& 1=\int_{0}^{\infty} f(R) \mathrm{d} R .
\end{aligned}
$$

The first equation can be integrated to find

$$
f(R)=C \exp \left(-\frac{(R-\widehat{R})^{2}}{2 \sigma^{2}}-a R\right),
$$

where

$$
\sigma=\sqrt{\frac{k_{\mathrm{B}} T}{8 \pi \gamma}}, a=\frac{4 q_{1}}{k_{\mathrm{B}} T}, C=\exp \left(k_{\mathrm{B}} T+q_{2}\right) .
$$

In principle, the constants $q_{1}, q_{2}$ can be determined by normalizing the distribution and imposing that the distribution averages to $\widehat{R}$. However, it is more useful to find an approximation. Since, we are interested in distributions close to an ideal particle size distribution, we assume that the width of the distribution $\sigma$ is much smaller than $R_{0}$ and introduce a small dimensionless parameter

$$
\epsilon=\frac{\sigma}{R_{0}}=\sqrt{\frac{k_{\mathrm{B}} T}{8 \pi \gamma R_{0}^{2}}}
$$

We expect $\widehat{R}$ to be close to $R_{0}$ so that we can replace the distribution $f(R)$ defined for positive $R$ by an approximation $f_{\text {app }}(R)$ defined for all real $R$, based on an expansion of the Gaussian obtained by expanding $f$. To lowest order, we have

$$
f_{\text {app }}(R)=\frac{1}{\sigma \sqrt{2 \pi}} e^{-\frac{(R-\widehat{R})^{2}}{2 \sigma^{2}}}\left(1+\mathcal{O}\left(a^{2}\right)\right)
$$

where the constants were determined by imposing that to order $\mathcal{O}\left(a^{2}\right)$,

$$
\int_{-\infty}^{+\infty} f_{\text {app }}(R) \mathrm{d} R=1, \quad \text { and } \quad \int_{-\infty}^{+\infty} f_{\text {app }}(R) R \mathrm{~d} R=\widehat{R} .
$$

By imposing conservation of total volume we find

$$
\widehat{R}=R_{0}\left(1-\epsilon^{2}\right) .
$$

We note that $\int_{-\infty}^{+\infty} f_{\text {app }}(R)(R-\widehat{R})^{3} \mathrm{~d} R=0$. Hence, our assumption (9) is automatically justified.

\section{REFERENCES}

[1] B. K. Chakraverty, J. Phys. Chem. Solids 28, 2401 (1967).

[2] B. L. V. Prasad, S. I. Stoeva, C. M. Sorensen, K. J. Klabunde, Chem. Mater. 15, 935 (2003).

[3] B. L. V. Prasad, S. I. Stoeva, C. M. Sorensen, K. J. Klabunde, Langmuir 18, 7515 (2002).

[4] A. B. Smetana, K. J. Klabunde, C. M. Sorensen, J. Colloid and Interface Sci. 284, 521 (2005).

[5] Pan Jun, A. O. El-Ballouli, L. Rollny, O. Voznyy, V. M. Burlakov, A. Goriely, E. H. Sargent, O. M. Bakr. ACS Nano 7, 10158 (2013).

[6] G. P. Dransfield, K. A. Fothergill, T. A. Egerton. In: Euro Ceramics (Vol. 1). Elsevier Applied Science Publishers, London, UK, 1989.

[7] S. R. Blackburn, T. A. Egerton, A. G. Jones, British Ceramics Proceedings 47, 87 (1991).

[8] A. N. Shipway, E. Katz, I. Willner, ChemPhysChem 1, 18 (2000).

[9] Sang-Hyun Yun, Seong Il Yoo, Jin Chul Jung, WangCheol Zin, Byeong-Hyeok Sohn, Chem. Mater. 18, 5646 (2006).

[10] Girish Muralidharan, Navakanta Bhat, Venugopal Santhanam, Nanoscale, 3, 4575 (2011).

[11] A. P. Alivisatos, Science, 271, 933 (1996).

[12] Xiaogang Peng, J. Wickham, A. P. Alivisatos, J. Am. Chem. Soc. 120, 5343 (1998).

[13] Shouheng Sun, C. B. Murray, Dieter Weller, Liesl Folks, Andreas Moser, Science 287, 1989 (2000).

[14] Jongham Park, Kwangjin An, Yosun Hwang, Je-Geun Park,Han-Jin Noh, Jae-Young Kim, Jae-Hoon Park, Nong-Moon Hwang, Taeghwan Hyeon, Nature Materials 3, 891 (2004).

[15] Z. Łodziana, Nan-Yu Topsøe, J. K. Nørskov, Nature Materials 3, 289 (2004).

[16] A. Mathur, P. Sharma, R. C. Cammarata, Nature Materials (correspondence) 4, 186 (2005).

[17] C. Z. Wagner, Electrochem. 65, 581 (1961). 
[18] J. Tersoff, AW Denier van der Gon, RM. Tromp, Phys. Rev. Lett. 70, 1143 (1993).

[19] D. Zhitomirsky, I. Kramer, AJ. Labelle, A. Fischer, R. Debnath, J. Pan, OM. Bakr, EH. Sargent, Nano letters, 12, 1007-1012 (2012).

[20] MS. Bootharaju, and VM. Burlakov, T. Besong, CP. Joshi, LG. AbdulHalim, DM. Black, RL Whetten, A. Goriely, OM. Bakr, Chemistry of Materials 27, 4289-4297 (2015).

[21] M. Baalousha, JR. Lead, Nature nanotechnology 8, 308209 (2013). 